\title{
Zollinger ellison syndrome due to primary nodal gastrinoma located outside the limits of the conventional gastrinoma triangle: report of a case
}

\author{
Ashish Arsia', Priya Hazrah ${ }^{1 *}$, Shabab Anwar ${ }^{1}$, Shaji Thomas ${ }^{1}$, Pooja Abbey ${ }^{2}$, Smita Singh $^{3}$
}

${ }^{1}$ Department of Surgery, Lady Hardinge Medical College, New Delhi, India
${ }^{2}$ Department of Radiology, Lady Hardinge Medical College, New Delhi, India
${ }^{3}$ Department of Pathology, Lady Hardinge Medical College, New Delhi, India

Received: 22 February 2021

Revised: 05 April 2021

Accepted: 14 April 2021

*Correspondence:

Dr. Priya Hazrah,

E-mail: priyahazrah@gmail.com

Copyright: (c) the author(s), publisher and licensee Medip Academy. This is an open-access article distributed under the terms of the Creative Commons Attribution Non-Commercial License, which permits unrestricted non-commercial use, distribution, and reproduction in any medium, provided the original work is properly cited.

\begin{abstract}
Primary nodal gastrinoma is a rare entity and the diagnosis is often contemplative when no other non - nodal primary site can be identified despite thorough investigations and operative exploration. Here we report one such case wherein a primary nodal gastrinoma was diagnosed as an entity of exclusion. Additionally, the location of the disease outside the confines of the conventional gastrinoma triangle further contributes to the rarity of the presentation. A young male patient had presented to us with history of multiple operations in the past for recurrent upper abdominal pain presumably consequential to peptic ulcer disease viz a trucal vagotomy and gastrojejunostomy, duodenal ulcer perforation surgery and a cholecystectomy. CT scan and endoscopic USG showed a preaortic calcified node located outside the limits of the gastrinoma triangle. A raised serum gastrin level and an endoscopic guided FNAC confirmed the diagnosis of a gastrinoma. A 68 Ga-DOTANOC PET CT revealed an exclusive nodal uptake with no discenable primary lesion. Normalization of gastrin levels after removal of the involved pre-aortic node further pointed to the diagnosis of primary nodal gastrinoma. A high index of clinical suspicion is warranted especially in a history of multiple surgeries for recurrent upper abdominal pain and location of the lesion outside the confines of the 'Gastrinoma Triangle' should not be deterrent for the diagnosis.
\end{abstract}

Keywords: Primary nodal gastrinoma, Zollinger ellison syndrome, Gastrinoma triangle

\section{INTRODUCTION}

Neuroendocrine tumors of the gastrointestinal tract are rare. Existence of primary nodal gastrinoma is rare and mired with controversies as to whether it is a primary nodal disease or a metastasis to a lymphnode from primary in the pancreas or gastrointestinal tract. ${ }^{1,2}$ They usually occur in the nests of embryonic tissue and lymph nodes in the region of gastrinoma triangle. Rarely the nodes may be located outside the Passaro triangle. ${ }^{3}$ Here we report one such case of gastrinoma with only discern- able nodal disease located outside the confines of the conventional gastrinoma triangle and discuss the relevant available literature.

\section{CASE REPORT}

A 35-year-old gentleman presented with history of severe upper abdominal pain, heartburn and loose stools for 9 months. The patient had undergone multiple surgeries in the past, for upper abdominal pain and peptic ulcer disease viz, truncal vagotomy and gastrojejunostomy 23 years back, operation for perforated duodenal ulcer 22 years 
back and laparoscopic cholecystectomy 12 years back. The patient had presented to us with the following investigations. An upper GI endoscopy showed the presence of a gastrojejunostomy with multiple ulcers and a polyp at anastomotic site and jejunal ulcers were noted.

A contrast enhanced CT Scan of the abdomen showed a well-defined rounded soft tissue density lesion with smooth margin and peripheral ring like calcification medially located in retro pancreatic location (separate from pancreas and great vessels), $23 \times 26 \mathrm{~mm}$ showing homogeneous post contrast enhancement. Another oval shaped non-calcified lesion measuring $20 \times 11 \mathrm{~mm}$ seen right of the lesion with possibility of being a lymphnode (Figure 1). An endoscopic ultrasound reiterated the CT findings and showed presence of two hyper echoic rounded lymph nodes with peripheral calcification largest measuring $26 \times 20 \mathrm{~mm}$. Fine needle aspiration cytology conducted under endoscopic USG guidance and was suggestive of neuroendocrine tumor. In 68 GaDOTANOC whole body PET CT an isodense, mildly contrast enhancing well defined lesion with minimal peripheral calcification and increased radiotracer uptake was noted in the preaortic location, at the D11-12 vertebral level (Figure 2). Also noted were two non-contrast enhancing sub centimetric lymph nodes in preaortic region medial to the lesion without radiotracer uptake. The serum biochemical tumor marker levels revealed a high serum gastrin levels with normal parathyroid hormone and calcium levels (serum gastrin= $1497 \mathrm{pg} / \mathrm{ml}$, normal range $=13-115 \mathrm{pg} / \mathrm{ml}$, serum parathyroid hormone $=47$ $\mathrm{pg} / \mathrm{ml}$, normal $=18-88 \mathrm{pg} / \mathrm{ml}$ and serum $\mathrm{Ca}=9.0 \mathrm{mg} / \mathrm{dl}$ ) Patient was taken up for elective exploratory laparotomy. Per operative findings were as follows: a lesion of $3 \times 2 \mathrm{~cm}$ was present $5 \mathrm{~cm}$ above the superior border of body of pancreas. The mass was excised and sent for frozen section which confirmed the possibility of it being a neuroendocrine tumor. One small adjacent node and another nodule were also excised and sent for HPE. There were no other lesion palpable in duodenum, pancreas, stomach and peri-aortic/aortocaval region. There were no operative complications and the postoperative recovery was uneventful. The final histopathology showed a lowgrade neuroendocrine tumor. Ki67 $<1 \%$, tumor was positive for nonspecific esterase and chromogranin.

One of the excised adjacent lymph-node showed reactive hyperplasia with sinus histiocytosis and the other showed foreign body giant cells along with fibrocollagenous tissue. Three months after the surgical excision of the mass the patient was advised an upper gastrointestinal endoscopy which revealed complete healing of ulcers at the gastrojejunostomy site as well as the jejunal ulcers and a snare polypectomy was done of the gastric polyp at the anastomotic site. Histopathology of the polyp showed it to be a hyperplastic polyp. Post-operative serum gastrin levels at 1 year follow up was normal $(96 \mathrm{pg} / \mathrm{ml})$. Patient is relieved of his symptoms and is currently not on any acid suppressive therapy.

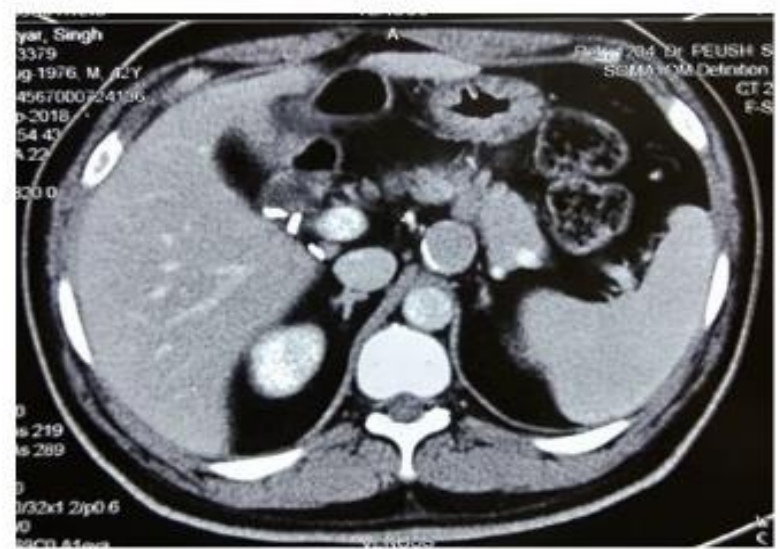

Figure 1: CECT showing well-defined rounded soft tissue density lesion with smooth margin and peripheral ring like calcification located in retro pancreatic location.

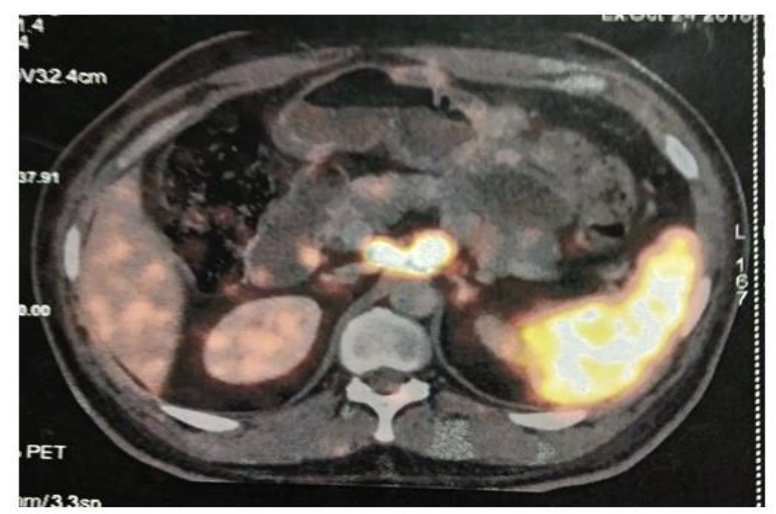

Figure 2: 68 Ga- DOTANOC whole body PET CT showed an isodense, mildly contrast enhancing well defined lesion with minimal peripheral calcification and increased radiotracer uptake in the preaortic location.

\section{DISCUSSION}

Zollinger Ellison syndrome is an endocrinopathy that presents with multiple recurrent and refractory peptic ulcers in the gastrointestinal tract due to hypersecretion of gastrin. Gastrinomas are rare neoplasia that may result in zollinger ellison syndrome and can occur sporadically or in association with multiple endocrine neoplasia (men 1). Our patient had undergone two surgeries related to peptic ulcer disease viz a truncal vagotomy and gastrojejunostomy 23 years back and an operation for duodenal ulcer perforation a year later. He also underwent a cholecystectomy for recurrent upper abdominal pain. His recent endoscopic finding of ulcers at gastrojejunostomy site as well as jejunal ulcers and raised gastrin levels were pathognomic of zollinger ellison syndrome.

It has been reported that in approximately $30 \%$ of sporadic gastrinomas localization with preoperative imaging and exploration may be negative as was in our case wherein the diagnosis and presumably localization of gastrinoma 
was elusive despite a long-protracted history of peptic ulcer disease and its complications. ${ }^{1}$ In addition to conventional methods of abdominal imaging, adjuncts like intraoperative USG, MRI intaroperative endoscopy and transillumination explorative duodenotomy have been advocated by authors to locate the primary and metastatic disease. ${ }^{1,2}$ Endoscopic USG has been found to be highly sensitive in pancreatic neuroendocrine tumors. ${ }^{4}$ The 68 Ga-DOTATATE PET/CT has been reported equivalent to a combination of SRS, MRI and thoraco-abdominopelvic CT for neuroendocrine tumors. ${ }^{5}$ In our case an abdominal CT and endoscopic USG showed a calcified lesion in the preaortic region. EUS guided FNAC were suggestive of neuroendocrine tumor and increased uptake in $68 \mathrm{Ga}$ DOTANOC PET/CT along with increased serum gastrin levels helped in further confirmation and localization of the disease. Incidence of of calcification in primary nodal gastrinoma is not known however calcification in pancreatic neuroendocrine tumors is rare and often associated in gastrinomas with aggressive disease particularly with lymph node and liver metastasis. ${ }^{6}$ In gastrinomas associated with men syndrome a possibility of metastatic calcification due to hyperparathyroidism may be postulated but in our patient the serum parathyroid and calcium were conspicuously normal ruling out the possibility of men syndrome.

The diagnosis of a primary nodal gastrinoma an ill-defined controversial entity is based upon findings of exclusively nodal disease particularly in the peripancreatic region mostly in the gastrinoma triangle and normalization of serum gastrin levels post resection. ${ }^{7-9}$ Primary nodal gastrinoma as a distinct clinical entity has been reported in literature and may be present in upto $10 \%$ of sporadic cases. $^{8,10-14}$ In a single institution study of 39 consecutive cases of gastrinoma 11 were found to be primary nodal gastrinoma. $^{7}$ In another retrospective study of extrapancreatic gastrinomas $2 / 23$ cases of extrapancreatic gastrinomas were nodal gastrinomas. ${ }^{14}$ The problem envisaged with diagnosis of a primary nodal gastrinoma is to differentiate it from a metastatic node from a primary elsewhere as a metastatic disease may portend a poorer outcome. ${ }^{1}$ Therefore, it is imperative to have a thorough preoperative investigation to look for primary and metastatic disease as was done in our case. The EUS and CT scan findings of an exclusive preaortic node as also the 68 Ga-DOTANOC PET/CT findings of exclusive uptake only in preaortic node were suggestive of the diagnosis of primary nodal gastrinoma as there were no other obvious primary site discernable. Normalization of serum gastrin levels post resection of the preaortic node further reiterated the diagnosis of existence of exclusive isolated nodal disease in the scenario and clinched the diagnosis. The presence of the inflammatory polyp in the stomach at the gastrojejunostomy site did raise a small probability initially of whether it could be a possible primary tumor, however the preoperative biopsy and non-uptake of radiotracer in 68 Ga-DOTANOC PET/CT and subsequently the final histopathology of snare polypectomy done ruled out possibility of it being a primary lesion and reconfirmed the diagnosis of a primary nodal disease.

It has been claimed that all nodal gastrinomas may be metastasis from occult gastrointestinal or hepatobiliary/pancreatic primary and rarely as primary nodal. ${ }^{8}$ It was observed that in a certain percentage of patients with primary nodal gastrinomas, duodenal microgastrinomas were detected in prolonged follow up (>10 years). ${ }^{13}$ Occult duodenal gastrinomas are associated with regional nodal metastasis in $60 \%$ cases and an aggressive approach has been advocated with systematic lymphadenectomy to extirpate the disease..$^{15}$ Occult submucosal gastrinomas have also been incidentally observed in patients being treated with gastrectomy as a method of gastric acid suppression in patients with hypergastrinema. ${ }^{16}$ Microgastrinomas have been incriminated as a cause for failed surgery in ZES. ${ }^{17}$ An explorative duodenotomy or intraoperative adjuncts like endoscopy and USG has been advocated by some authors. ${ }^{2}$ The significance of routine use of duodenotomy to detect duodenal microgastrinomas as proposed earlier needs to be re-evaluated, in an era of $68 \mathrm{Ga-DOTANOC} \mathrm{PET/CT} \mathrm{as}$ most authors advocating explorative duodenotomy had not investigated its use in their study. 2,10,17

Despite the above existing deterrents primary nodal gastrinomas exist as a distinct entity and may not necessarily be a metastatsis from an occult primary. Histopathological studies have shown the rare $(<1 \%)$ presence of neuroendocrine cells in the lymph nodes of the peripancreatic region thus substantiating the possibility for the origin of an exclusive primary nodal gastrinoma, even though their potential to secrete hormones is not known. ${ }^{18}$ Another point of contention that needs to be noted in this regard is that unlike metastatic nodal gastrinoma with a primary elsewhere wherein a poor disease free survival is portended, a primary nodal gastrinoma without any evidence of disease elsewhere viz the gastrointestinal hepatobiliary or pancreatic region have been reported to have a good prognosis and disease free survival to the extent of 18 years have been documented. ${ }^{8,15,19,20}$ Primary nodal gastrinomas often do well after isolated excision of the node and more extensive procedures like pancreaticoduodenectomy is not deemed necessary in view of the long survival benefits portrayed with limited procedures and high morbidity of extensive resection. ${ }^{2,12}$ A 23-year history is noted in our patient of having undergone treatment for peptic ulcer disease and its complication is presumed to be due to the gastrinoma which evaded detection. The long history and low-grade histopathology of the lesion further reiterates the indolent nature of the disease in our case and validates the diagnosis of primary nodal gastrinoma rather than metastasis to lymph node from a gastrinoma elsewhere in the gastrointestinal tract wherein a higher grade tumor and much poorer outcome would have been anticipated as discussed above. 
Most occult gastrinomas have been reported to be located within the confines of the boundaries of gastrinoma triangle defined as follows : superiorly by the junction of the cystic and common bile ducts, inferiorly the junction of the second and third portions of the duodenum, and the junction of the neck and body of the pancreas medially. ${ }^{21}$ Nevertheless, the primary tumor in a gastrinoma can be in the duodenum, pancreas, lymph nodes, hepatobiliary tract or rarely in ovaries heart omentum and jejunum. ${ }^{1}$ Primary nodal gastrinomas have been classically defined to be located within the confines of the well-defined 'gastrinoma triangle'. ${ }^{19-21}$ Extrapancreatic gastrinomas outside the confines of the above triangle has been rarely reported. ${ }^{3}$ In our patient the node was located in the peripancreatic region but outside the limits of boundaries of the defined gastrinoma triangle adding to the further rarity of the presentation.

\section{CONCLUSION}

Primary nodal gastrinomas may present de novo as isolated nodal disease in the peripancreatic region. Its location outside the confines of the gastrinoma triangle should not be a deterrent in the diagnosis. A thorough preoperative workup with advanced imaging techniques like endoscopic USG and 68 Ga-DOTANOC PET/CT aids the diagnosis. Limited resection of the nodal disease can lead to regression of symptoms and help achieve eugastrinemic levels. Primary nodal gastrinomas have a better outcome than metastatic nodal disease from an occult gastrinoma.

\section{ACKNOWLEDGEMENTS}

We are thankful to the department of surgery, radiology and pathology for their support.

Funding: No funding sources

Conflict of interest: None declared

Ethical approval: Not required

\section{REFERENCES}

1. Shao QQ, Zhao BB, Dong LB, Cao HT, Wang WB. Surgical management of Zollinger-Ellison syndrome: Classical considerations and current controversies. World J Gastroenterol. 2019 Aug 28;25(32):4673-81.

2. Arnold WS, Fraker DL, Alexander HR, Weber HC, Norton JA, Jensen RT. Apparent lymph node primary gastrinoma. Surgery. 1994 ;116(6):1123-9.

3. Toppino M, Corno F, Serra GC, Mistrangelo M, Guglielminotti P, Bolla C, et al. Extrahepatic gastrinoma. Report of a clinical case. Minerva Gastroenterol Dietol. 1996;42(3):161-7.

4. Anderson MA, Carpenter S, Thompson NW, Nostrant TT, Elta GH, Scheiman JM. Endoscopic ultrasound is highly accurate and directs management in patients with neuroendocrine tumors of the pancreas. Am J Gastroenterol. 2000;95(9):2271-7.

5. Gabriel S, Garrigue P, Dahan L, Castinetti F, Sebag F, Baumstark K, et al. Prospective evaluation of 68 Ga-DOTATATE PET/CT in limited disease neuroendocrine tumours and/or elevated serum neuroendocrine biomarkers. Clin Endocrinol (Oxf). 2018;89(2):155-63.

6. Poultsides GA, Huang LC, Chen Y, Visser BC, Pai RK, Jeffrey RB, et al. Pancreatic neuroendocrine tumors: radiographic calcifications correlate with grade and metastasis. Ann Surg Oncol. 2012;19(7):2295-303.

7. Chen Y, Deshpande V, Ferrone C, Blaszkowsky LS, Parangi S, Warshaw AL, et al. Primary lymph node gastrinoma: A single institution experience. Surgery. 2017;162(5):1088-94.

8. Delcore R, Cheung LY, Friesen SR. Outcome of lymph node involvement in patients with the Zollinger-Ellison syndrome. Ann Surg. 1988;208(3):291-8.

9. Bhagavan BS, Slavin RE, Goldberg J, Rao RN. Ectopic gastrinoma and Zollinger-Ellison syndrome. Hum Pathol. 1986;17(6):584-92.

10. Norton JA, Alexander HR, Fraker DL, Venzon DJ, Gibril F, Jensen RT. Possible primary lymph node gastrinoma: occurrence, natural history, and predictive factors: a prospective study. Ann Surg. 2003;237(5):650-7.

11. Odelowo OO, Nidiry JJ, Zulu SH. Primary lymph node gastrinoma: a case report. J Natl Med Assoc. 2003;95(2):168-71.

12. Jaenigen B, Kayser G, Steinke B, Thomusch O. FiveYear Long-Term Followup of a Primary Lymph node Gastrinoma: Is a Pancreaticoduodenectomy Justified? Case Rep Med. 2009;2009:762791.

13. Harper S, Carroll RW, Frilling A, Wickremesekera SK, Bann S. Primary lymph node gastrinoma: 2 cases and a review of the literature. J Gastrointest Surg. 2015;19(4):651-5.

14. Farley DR, van Heerden JA, Grant CS, Thompson GB. Extrapancreatic gastrinomas. Surgical experience. Arch Surg. 1994;129(5):506-11.

15. Zogakis TG, Gibril F, Libutti SK, Norton JA, White DE, Jensen RT, et al. Management and outcome of patients with sporadic gastrinoma arising in the duodenum. Ann Surg. 2003;238(1):42-8.

16. Delcore R, Friesen SR. Zollinger-Ellison syndrome. A new look at regression of gastrinomas. Arch Surg. 1991;126(5):556-8.

17. Thompson NW, Vinik AI, Eckhauser FE. Microgastrinomas of the duodenum. A cause of failed operations for the Zollinger-Ellison syndrome. Ann Surg. 1989;209(4):396-404.

18. Perrier ND, Batts KP, Thompson GB, Grant CS, Plummer TB. An immunohistochemical survey for neuroendocrine cells in regional pancreatic lymph nodes: a plausible explanation for primary nodal gastrinomas? Mayo Clinic Pancreatic Surgery Group. Surgery. 1995;118(6):957-65. 
19. Herrmann ME, Ciesla MC, Chejfec G, DeJong SA, Yong SL. Primary nodal gastrinomas. Arch Pathol Lab Med. 2000;124(6):832-5.

20. Singh D, Lal SB, Sood A, Gupta R, Kumar R, Vashishta RK, et al. Management of Primary Lymph Nodal Gastrinoma With Liver Metastases Resulting in Zollinger-Ellison Syndrome. Clin Nucl Med. 2019;44(1):36-9.
21. Stabile BE, Morrow DJ, Passaro E Jr. The gastrinoma triangle: operative implications. Am J Surg. 1984;147(1):25-31.

Cite this article as: Arsia A, Hazrah P, Anwar S, Thomas S, Abbey P, Singh S. Zollinger ellison syndrome due to primary nodal gastrinoma located outside the limits of the conventional gastrinoma triangle: report of a case. Int Surg J 2021;8:1653-7. 